\title{
Constructing Carrollian CFTs
}

\section{Nishant Gupta and Nemani V. Suryanarayana}

Institute of Mathematical Sciences, Taramani, Chennai 600 113, India

Homi Bhabha National Institute, Anushakti Nagar, Mumbai 400085, India

E-mail: nishantg@imsc.res.in, nemani@imsc.res.in

ABSTRACT: We construct classical theories for scalar fields in arbitrary Carroll spacetimes that are invariant under Carrollian diffeomorphisms and Weyl transformations. When the local symmetries are gauge fixed these theories become Carrollian conformal field theories. We show that generically there are at least two types of such theories: one in which only time derivatives of the fields appear and the other in which both space and time derivatives appear. A classification of such scalar field theories in three (and higher) dimensions up to two derivative order is provided. We show that only a special case of our theories arises in the ultra-relativistic limit of a covariant parent theory.

Keywords: Conformal Field Theory, Gauge-gravity correspondence, Space-Time Symmetries

ARXIV EPRINT: 2001.03056 


\section{Contents}

1 Introduction 1

2 Conformally coupled scalar field - revisited 3

3 Scalar field theories on Carroll spacetimes 5

3.1 Constructing equations of motion 6

$\begin{array}{ll}3.2 \text { Constructing actions } & 10\end{array}$

4 A Carroll CFT from a conformally coupled scalar $\quad 14$

5 Gauge fixing and residual symmetries $\quad 15$

6 Conclusion 18

A Extension to general dimensions - some details $\quad 19$

\section{Introduction}

The Poincare algebra admits two interesting limiting algebras obtained by Inonu-Wigner contraction where one takes the speed of light $c \in[0, \infty)$ to either zero or infinity. When $c \rightarrow \infty$ one obtains the well-known Galilean algebra, and when $c \rightarrow 0$ one ends up with the so-called Carrollian algebra [1,2]. The former case has played a very important role as it is relevant for a host of physical systems in which typical velocities involved are very small compared to the speed of light, such as Newtonian mechanics, many condensed matter systems etc. The Carroll limit is relevant if velocities involved are all very close to $c$ and it remained unexplored for a long time until the last decade.

An important realisation of the Poincare algebra arises from the isometries of the flat Minkowski spacetime with the standard line element:

$$
d s^{2}=-c^{2} d t^{2}+d \mathbf{x} \cdot d \mathbf{x}
$$

which is an example of a pseudo-Riemannian manifold, with non-degenerate Lorentzian metric $g_{\mu \nu}=\eta_{\mu \nu}$. If one takes either of the limits $c \rightarrow \infty$ (better done on $g^{\mu \nu}$ ) or $c \rightarrow 0$ (done on $g_{\mu \nu}$ ) one obtains a manifold with a degenerate metric tensor. The geometries that realise Galilean or Carrollian algebras are therefore not Riemannian manifolds but in fact belong to a more general class of geometrical objects called Newton-Cartan manifolds (for $c \rightarrow \infty$ case) and Carroll manifolds (for $c \rightarrow 0$ case) respectively (see, for instance, [3]).

The conformal algebra contains the Poincare algebra as a subalgebra, and also plays an important role in physics as it is relevant in many contexts, such as: symmetry algebra 
of theories at the fixed points of RG flows, AdS/CFT etc. Hence the Galilei and Carroll limits of the Conformal algebra have also been studied in the past.

Just as the conformal algebra can be represented by conformal Killing vectors (CKV) of (conformally) flat spacetimes, such as $\mathbb{R} \times S^{d}$ with line element

$$
d s^{2}=-c^{2} d t^{2}+d \Omega_{d}^{2}
$$

or $\mathbb{R}^{1, d}$, the Galilean and Carrollian conformal algebras can be thought of as appropriately defined conformal algebras of (conformally) flat Galilei and Carroll spacetimes. The conformal algebra of (any spacetime conformal to) Minkowski spacetime $M_{d+1}$ is finite dimensional for $d \geq 2$ and therefore obtaining the corresponding Galilean or Carrollian conformal algebras via Inonu-Wigner contraction will necessarily result in finite dimensional algebras for $d \geq 2$. However, if one defines these algebras directly as conformal algebras of the corresponding Galilei and Carroll spacetimes one may get bigger algebras than those obtained by the contraction procedure. In fact there is a more general notion of conformal symmetry even in the flat Carroll spacetime $C_{d+1}$, parametrised by $z$, the analog of the dynamical exponent of Galilean conformal transformations, which in turn is given by a non-negative integer $k$ by $z=2 / k$ in [4]. One obtains only the $z=1(k=2)$ case via the contraction procedure.

Also as was shown in [5] the Carrollian conformal algebra $\mathfrak{c c a}_{d+1}^{(z)}$ for $d=2$ and $k=2$ is the $\mathfrak{b m s}_{4}$ algebra $[7,8]$, which is infinite dimensional. This is because the asymptotic null boundary $\mathcal{I}^{ \pm}$of flat spacetime with metric

$$
d s^{2}=-0 \times d t^{2}+d \Omega_{d}^{2}
$$

is a Carroll manifold. It was well-known that the asymptotic symmetry algebra of 4dimensional gravity (without cosmological constant) is the famous $\mathfrak{b m s}_{4}$ algebra $[7,8]$.

So if one wants to describe some $(d+2)$-dimensional gravitational theory with asymptotically flat boundary conditions holographically then the holograms should be field theories on $\mathcal{I}^{ \pm}$with $\mathfrak{c c a}_{d+1}^{(z=1)}$ as their global symmetries [9, 10]. This has led to a lot of work seeking field theories on null-manifolds which are Carrollian CFTs (see for example [3$6,11-17])$. The procedure followed there is typically to start with a CFT on $M_{d+1}$ and take an ultra-relativistic limit. This has been a fruitful exercise and has resulted in quite a lot of interesting examples with $\mathfrak{c c a}_{d+1}^{(z=1)}$ symmetries.

It is expected that constructing field theories directly on a Carroll manifold $\mathcal{C}_{d+1}$ to give rise to more general class of theories than those obtained by taking ultra-relativistic limit of known CFTs on the parent pseudo-Riemannian manifold $\mathcal{M}_{d+1}$. The main aim of this paper is to demonstrate that this expectation is indeed realised. We will construct classical scalar field theories on generic 3-dimensional Carroll manifolds that are invariant under both diffeomorphisms and Weyl transformations of the Carroll manifold. These theories can be put on any given Carroll manifold - of which the interesting examples include null boundaries of asymptotically flat spacetimes, horizons of black holes, boundaries of causal developments, etc. Then the residual symmetries should, by construction, make the resultant theory have $\mathfrak{c c a}_{3}^{(z)}$ as the symmetry algebra for generic values of $z$. We will 
mainly concentrate on $d=2$ for most part (relegating the higher dimensional case to an appendix). We obtain a larger class of theories for scalar fields going beyond what one obtains by the method of taking ultra-relativistic limit.

Summary of results. On a generic 3-dimensional Carroll manifold we have constructed diffeomorphism and Weyl covariant equations of motion as well as invariant actions (when they exist) of a scalar field $\Phi(t, \mathbf{x})$ up to second order derivatives in both time $(t)$ and space $(\mathbf{x})$ coordinates, for general values of $z$ and the conformal dimension $\delta$ of $\Phi(t, \mathbf{x})$. Our results include:

- Two classes of diffeomorphic and Weyl covariant equations of motion, one with two time-derivatives of the field, and the other with (up to) two space-derivatives. These exist for general values of $z$ and $\delta$.

- For the special value $z=1$ there is one equation of motion with at least five real parameters.

- For $z=2$ there are two classes of equations of motion: one with second order timederivatives and the other with first order time- and second order space-derivatives.

- The invariant actions exist only when $\delta$ is restricted to either $\delta=\frac{z}{2}$ or $\delta=1-\frac{z}{2}$.

- Having a stable monomial potential $\left(\Phi^{2 n}\right)$ further restricts the values of $z$ to be determined in terms of the degree of the monomial.

- Gauge fixing the local symmetries in an appropriate way leads to field theories with Carrollian conformal algebra $\mathfrak{c c a}_{3}^{(z)}$ worth of symmetries that have an additional fluctuating two-component field along with the scalar field.

The rest of the paper is organised as follows. The section 2 contains a review of how one constructs a diffeomorphic and Weyl invariant theory of a scalar field in the background of a generic (pseudo) Riemann manifold. In section 3 we turn to repeating the steps of section 2 to the case of scalars in a Carroll manifold. There we present the details of our construction of equations of motion as well as actions. In section 4 we show how a subset of results of section 3 can be recovered starting from the conformally coupled scalar with the background metric in Randers-Papapetrou form. In section 5 we gauge fix our theories to recover and generalise the classical scalar field theories with $\mathfrak{c c a}_{3}^{(z)}$ algebra worth of symmetries. We conclude with some remarks and open questions in section 6 . The appendix A contains some details of the case of arbitrary dimensions.

\section{Conformally coupled scalar field - revisited}

In this section we (re) construct the well-known conformally coupled scalar field equation of motion and its action in detail. This will provide us with the method we follow in the later sections. The result we want to re-derive is the diffeomorphic and Weyl invariant 
classical (free) scalar field theory in $d=2+1$ dimensions in a general background with metric $g_{\mu \nu}$. This has the action:

$$
\mathcal{L}=-\frac{1}{2} \sqrt{-g}\left(g^{\mu \nu} \partial_{\mu} \phi \partial_{\nu} \phi+\frac{1}{8} R \phi^{2}\right)
$$

and the equation of motion

$$
g^{\mu \nu} \nabla_{\mu} \nabla_{\nu} \phi-\frac{1}{8} R \phi=0 .
$$

This action (2.1) is invariant and the equation of motion (2.2) is covariant under the Weyl transformations:

$$
g_{\mu \nu}^{\prime}(x)=\frac{1}{B^{2}} g_{\mu \nu}(x), \quad \phi^{\prime}(x)=B^{\frac{1}{2}} \phi(x)
$$

where $B$ is an arbitrary function of the coordinates, and diffeomorphisms:

$$
g_{\mu \nu}^{\prime}\left(x^{\prime}\right)=\frac{d x^{\alpha}}{d x^{\prime \mu}} \frac{d x^{\beta}}{d x^{\prime \nu}} g_{\alpha \beta}(x), \quad \phi^{\prime}\left(x^{\prime}\right)=\phi(x)
$$

where $x \rightarrow x^{\prime \mu}(x)$ is a coordinate transformation. Let us rederive this result (see Wald [18] for instance). For this one starts with scalar (under diffeomorphisms in (2.4)) combinations that are linear in $\Phi$ and have (at most) two derivatives, namely, $R \Phi$ and $\nabla_{\mu} \nabla^{\mu} \Phi$. Their transformation properties under the Weyl transformations $g_{\mu \nu} \rightarrow B^{-2} g_{\mu \nu}$ and $\Phi \rightarrow B^{\delta} \Phi$, are

$$
\begin{aligned}
R \Phi \longrightarrow B^{\delta} & {\left[B^{2} R+4 B g^{\mu \nu} \nabla_{\mu} \nabla_{\nu} B-6 g^{\mu \nu} \nabla_{\mu} B \nabla_{\nu} B\right] \Phi } \\
g^{\mu \nu} \nabla_{\mu} \nabla_{\nu} \Phi \longrightarrow B^{\delta}[ & B^{2} g^{\mu \nu} \nabla_{\mu} \nabla_{\nu} \Phi+\delta \Phi B g^{\mu \nu} \nabla_{\mu} \nabla_{\nu} B+\delta(\delta-2) \Phi g^{\mu \nu} \nabla_{\mu} B \nabla_{\nu} B \\
& \left.\quad(2 \delta-1) B g^{\mu \nu} \nabla_{\mu} B \nabla_{\nu} \Phi\right]
\end{aligned}
$$

where $\delta$ is the Weyl weight of the scalar $\Phi$. For a linear combination of $R \Phi$ and $\square \Phi$ to be covariant all the inhomogeneous terms in the Weyl transformation of that combination should cancel out. But in no linear combination the term containing $B g^{\mu \nu} \nabla_{\mu} B \nabla_{\nu} \Phi$ on the right hand side of $\square \Phi$ in (2.5) gets canceled. So its coefficient $(2 \delta-1)$ has to vanish identically, giving us $\delta=\frac{1}{2}$. Then the only linear combination that transforms homogeneously is

$$
g^{\mu \nu} \nabla_{\mu} \nabla_{\nu} \Phi-\frac{1}{8} R \Phi \rightarrow B^{\frac{5}{2}}\left[g^{\mu \nu} \nabla_{\mu} \nabla_{\nu} \Phi-\frac{1}{8} R \Phi\right]
$$

showing that the equation (2.2) is the only covariant one. For the construction of an action one notes:

$$
g^{\mu \nu} \nabla_{\mu} \Phi \nabla_{\nu} \Phi \longrightarrow B\left[B^{2} g^{\mu \nu} \nabla_{\mu} \Phi \nabla_{\nu} \Phi+\frac{1}{4} \Phi^{2} g^{\mu \nu} \nabla_{\mu} B \nabla_{\nu} B+\Phi B g^{\mu \nu} \nabla_{\mu} \Phi \nabla_{\nu} B\right]
$$

which can be used along with the first line of (2.5) to show that

$$
\sqrt{g}\left[g^{\mu \nu} \nabla_{\mu} \Phi \nabla_{\nu} \Phi+\frac{1}{8} R \Phi^{2}\right] \longrightarrow \sqrt{g}\left[g^{\mu \nu} \nabla_{\mu} \Phi \nabla_{\nu} \Phi+\frac{1}{8} R \Phi^{2}\right]+\partial_{\mu}\left[\frac{\Phi^{2}}{2 B} \sqrt{g} g^{\mu \nu} \partial_{\nu} B\right] \text {. }
$$

So the action (2.1) is also invariant under (2.3), (2.4) and results in the equation of motion (2.2). In the next section we use the same procedure to construct analogous equations of motion and actions for scalar fields on 3-dimensional Carroll manifolds. 


\section{Scalar field theories on Carroll spacetimes}

Let us first review some essential aspects of Carrollian geometries. We will follow notations and conventions of Ciambelli et al. $[11,13]$ here. A Carroll spacetime is a fibre bundle $\mathcal{C}_{d+1}$ with a $d$-dimensional base $\mathcal{S}$ and one-dimensional fibre. We work with local coordinates $\mathbf{x}$ on the base and $t$ on the fibre. Then the Carroll spacetime is specified by a non-degenerate $d$-dimensional metric $a_{i j}(t, \mathbf{x})$ on the base $\mathcal{S}$, the Ehresmann connection 1-form $b_{i}(t, \mathbf{x})$ and a scalar $\omega(t, \mathbf{x})$. Then one defines the Carroll diffeomorphisms as those that keep this structure invariant. In our coordinates they take the form:

$$
t \rightarrow t^{\prime}(t, \mathbf{x}), \quad \mathbf{x} \rightarrow \mathbf{x}^{\prime}(\mathbf{x})
$$

The Jacobian of these Carroll diffeomorphisms $\left(t, x^{i}\right) \rightarrow\left(t^{\prime}(t, \mathbf{x}), x^{\prime i}(\mathbf{x})\right)$ is the matrix

$$
\left(\begin{array}{cc}
J(t, \mathbf{x}) & J_{i}(t, \mathbf{x}) \\
0 & J^{j}{ }_{i}
\end{array}\right)
$$

where $J=\frac{\partial t^{\prime}}{\partial t}, J_{i}=\frac{\partial t^{\prime}}{\partial x^{i}}$, and $J^{j}{ }_{i}=\frac{\partial x^{\prime j}}{\partial x^{i}}$, with its inverse

$$
\left(\begin{array}{cc}
J^{-1} & -J^{-1} J_{k}\left(J^{-1}\right)^{k}{ }_{i} \\
0 & \left(J^{-1}\right)^{j}{ }_{i}
\end{array}\right)
$$

where $\left(J^{-1}\right)^{i}{ }_{j}$ is the inverse of the matrix $J^{i}{ }_{j}$. Under these transformations the geometrical data $\left(a_{i j}, b_{i}, \omega\right)$ of the Carroll spacetime transforms as:

$$
\begin{aligned}
a_{i j}^{\prime}\left(t^{\prime}, \mathbf{x}^{\prime}\right) & =a_{k l}(t, \mathbf{x})\left(J^{-1}\right)^{k}{ }_{i}\left(J^{-1}\right)_{j}^{l}, \quad \omega^{\prime}\left(t^{\prime}, \mathbf{x}^{\prime}\right)=J^{-1} \omega(t, \mathbf{x}) \\
b_{k}^{\prime}\left(t^{\prime}, \mathbf{x}^{\prime}\right) & =\left(b_{i}(t, \mathbf{x})+J^{-1} J_{i} \omega(t, \mathbf{x})\right)\left(J^{-1}\right)^{i}{ }_{k}
\end{aligned}
$$

along with $\partial_{t}^{\prime}=J^{-1} \partial_{t}, \partial_{j}^{\prime}=\left(J^{-1}\right)^{i}{ }_{j}\left(\partial_{i}-J^{-1} J_{i} \partial_{t}\right)$. Now one can list the objects that are covariant under the Carroll diffeomorphisms. At the first derivative order one has

$$
\phi_{i}=\frac{1}{\omega}\left(\partial_{i} \omega+\partial_{t} b_{i}\right), \quad \hat{\gamma}_{j}^{i}=\frac{1}{2 \omega} a^{i k} \partial_{t} a_{j k}, \quad \theta=\frac{1}{\omega} \partial_{t} \ln \sqrt{a}=\hat{\gamma}_{i}^{i}, \quad f_{i j}=2\left(\partial_{[i} b_{j]}+b_{[i} \phi_{j]}\right) .
$$

Because these are covariant one can raise and lower the indices using $a_{i j}$ and its inverse $a^{i j}$. One also has the following differential operators

$$
\hat{\partial}_{t}=\frac{1}{\omega} \partial_{t}, \quad \hat{\partial}_{i}=\partial_{i}+\frac{b_{i}}{\omega} \partial_{t}
$$

that are covariant. Then the Carroll-Christoffel connection

$$
\hat{\gamma}_{j k}^{i}=\frac{1}{2} a^{i l}\left(\hat{\partial}_{j} a_{l k}+\hat{\partial}_{k} a_{j l}-\hat{\partial}_{l} a_{j k}\right)
$$

allows one to write down further sets of covariant objects. This connection transforms under Carroll diffeomorphisms in the same manner as the usual Christoffel connection in Riemannian geometry. We will define the Carroll tensors to transform as:

$$
\Phi^{\prime}=\Phi, \quad V^{\prime i}=J_{j}^{i} V^{j}, \quad V_{i}^{\prime}=V_{j}\left(J^{-1}\right)_{i}^{j}, \quad \text { etc. }
$$


Under the Carroll connection (3.7) $a_{i j}$ and $a^{i j}$ are covariantly constant. Another fact is that if one defines $\hat{\partial}_{t}:=\frac{1}{\omega} \partial_{t}$ and $\hat{\nabla}_{t} a_{i j}:=\hat{\partial}_{t} a_{i j}-\hat{\gamma}_{i}^{k} a_{k j}-\hat{\gamma}_{j}^{k} a_{i k}$ and $\hat{\nabla}_{t} a^{i j}:=\hat{\partial}_{t} a^{i j}+\hat{\gamma}_{k}^{i} a^{k j}+\hat{\gamma}_{k}^{j} a^{i k}$ then the metric $a_{i j}$ and its inverse are covariantly constants under $\hat{\nabla}_{t}$ as well.

At the second derivative order one can list the following invariant objects:

$$
\theta^{2}, \quad \hat{\partial}_{t} \theta=\frac{1}{\omega} \partial_{t} \theta, \quad \hat{\gamma}_{j}^{i} \hat{\gamma}_{i}^{j}, \quad \hat{r}, \quad a^{i j} \hat{\nabla}_{i} \phi_{j}, \quad a^{i j} \phi_{i} \phi_{j}
$$

where $\hat{r}$ is the Carroll Ricci scalar defined [13] as follows:

$$
\hat{r}_{j k l}^{i}=\hat{\partial}_{k} \hat{\gamma}_{l j}^{i}-\hat{\partial}_{l} \hat{\gamma}_{k j}^{i}+\hat{\gamma}_{k m}^{i} \hat{\gamma}_{l j}^{m}-\hat{\gamma}_{l m}^{i} \hat{\gamma}_{k j}^{m}, \quad \hat{r}_{i j}=\hat{r}_{i k j}^{k}, \quad \hat{r}=a^{i j} \hat{r}_{i j} .
$$

Next we consider Carroll Weyl transformations. Following [11] we define this as

$\tilde{a}_{i j}(t, \mathbf{x})=(B(t, \mathbf{x}))^{-2} a_{i j}(t, \mathbf{x}), \quad \tilde{\omega}(t, \mathbf{x})=(B(t, \mathbf{x}))^{-z} \omega(t, \mathbf{x}), \quad \tilde{b}_{i}(t, \mathbf{x})=(B(t, \mathbf{x}))^{-z} b_{i}(t, \mathbf{x})$

where $B(t, \mathbf{x})$ is an arbitrary function and $z$ is a non-zero real number. We are now ready to emulate the steps of section 2 and construct equations of motion that are covariant under the Carroll diffeomorphisms (3.1) and Weyl transformations (3.11).

\subsection{Constructing equations of motion}

For this we first start by listing the transformation properties of our Carroll diffeomorphism invariants (3.9) under (3.11). One finds:

$$
\begin{aligned}
\theta & \longrightarrow B^{z-1}\left[B \theta-2 \hat{\partial}_{t} B\right], \\
\hat{\partial}_{t} \theta & \longrightarrow B^{2 z-2}\left[B^{2} \hat{\partial}_{t} \theta+z B \theta \hat{\partial}_{t} B-2(z-1)\left(\hat{\partial}_{t} B\right)^{2}-2 B \hat{\partial}_{t} \hat{\partial}_{t} B\right], \\
\hat{\gamma}_{j}^{i} \hat{\gamma}_{i}^{j} & \longrightarrow B^{2 z-2}\left[B^{2} \hat{\gamma}_{j}^{i} \hat{\gamma}_{i}^{j}-2 B \theta \hat{\partial}_{t} B+2\left(\hat{\partial}_{t} B\right)^{2}\right], \\
\hat{r} & \longrightarrow B^{2} \hat{r}+2 B a^{i j} \hat{\nabla}_{i} \hat{\partial}_{j} B-2 a^{i j} \hat{\partial}_{i} B \hat{\partial}_{j} B, \\
a^{i j} \hat{\nabla}_{i} \phi_{j} & \longrightarrow B^{2} a^{i j} \hat{\nabla}_{i} \phi_{j}-z B a^{i j} \hat{\nabla}_{i} \hat{\partial}_{j} B+z a^{i j} \hat{\partial}_{i} B \hat{\partial}_{j} B \\
a^{i j} \phi_{i} \phi_{j} & \longrightarrow B^{2} a^{i j} \phi_{i} \phi_{j}-2 z B \phi^{i} \hat{\partial}_{i} B+z^{2} a^{i j} \hat{\partial}_{i} B \hat{\partial}_{j} B .
\end{aligned}
$$

There are three combinations that transform homogeneously:

$$
\begin{aligned}
\hat{r}+\frac{2}{z} a^{i j} \hat{\nabla}_{i} \phi_{j} & \longrightarrow B^{2}\left(\hat{r}+\frac{2}{z} a^{i j} \hat{\nabla}_{i} \phi_{j}\right), \\
\hat{\gamma}_{j}^{i} \hat{\gamma}_{i}^{j}-\frac{1}{2} \theta^{2} & \longrightarrow B^{2 z}\left(\hat{\gamma}_{j}^{i} \hat{\gamma}_{i}^{j}-\frac{1}{2} \theta^{2}\right), \\
f_{i j} f^{i j} & \longrightarrow B^{4-2 z} f_{i j} f^{i j} .
\end{aligned}
$$

Now we are ready to include matter fields. Let us consider a real scalar field $\Phi(t, \mathbf{x})$ for simplicity. We seek to construct equations of motion which are Carroll Weyl invariant. For this we start by listing Carroll diffeomorphism invariants up to two derivatives (on both sets of objects $\left(a_{i j}, b_{i}, \omega\right)$ and $\left.\Phi\right)$, and linear in $\Phi$ :

$\Phi, \quad \theta \Phi, \quad \theta^{2} \Phi, \quad \hat{\gamma}_{j}^{i} \hat{\gamma}_{i}^{j} \Phi, \quad \hat{\partial}_{t} \theta \Phi, \quad \hat{r} \Phi, \quad \hat{\nabla}^{i} \phi_{i} \Phi, \quad \phi^{i} \phi_{i} \Phi, \quad f_{i j} f^{i j} \Phi$

$\hat{\partial}_{t} \Phi, \quad \theta \hat{\partial}_{t} \Phi, \quad \phi^{i} \hat{\partial}_{i} \Phi, \quad \hat{\partial}_{t} \hat{\partial}_{t} \Phi, \quad \hat{\nabla}^{i} \hat{\partial}_{i} \Phi$ 
Defining that the scalar $\Phi$ transforms as $\Phi \rightarrow B^{\delta} \Phi$ under Weyl transformations we can find how the objects in (3.14) above transform. We find:

$$
\begin{aligned}
\hat{\partial}_{t} \Phi & \longrightarrow B^{z+\delta-1}\left[B \hat{\partial}_{t} \Phi+\delta \Phi \hat{\partial}_{t} B\right] \\
\hat{\partial}_{t} \hat{\partial}_{t} \Phi & \longrightarrow B^{\delta+2 z-2}\left[B^{2} \hat{\partial}_{t} \hat{\partial}_{t} \Phi+(z+2 \delta) B \partial_{t} B \partial_{t} \Phi+\delta B \Phi \hat{\partial}_{t} \hat{\partial}_{t} B+\delta(\delta+z-1) \Phi\left(\hat{\partial}_{t} B\right)^{2}\right], \\
\phi^{i} \hat{\partial}_{i} \Phi & \longrightarrow B^{\delta}\left[B^{2} \phi^{i} \hat{\partial}_{i} \Phi+\delta B \Phi \phi^{i} \hat{\partial}_{i} B-\delta z \Phi \hat{\partial}_{i} B \hat{\partial}^{i} B-z B \hat{\partial}^{i} \Phi \hat{\partial}_{i} B\right] \\
\hat{\nabla}^{i} \hat{\partial}_{i} \Phi & \longrightarrow B^{\delta}\left[B^{2} \hat{\nabla}^{i} \hat{\partial}_{i} \Phi+\delta(\delta-1) \Phi \hat{\partial}^{i} B \hat{\partial}_{i} B+2 \delta B \hat{\partial}^{i} B \hat{\partial}_{i} \Phi+\delta B \hat{\nabla}^{i} \hat{\partial}_{i} B\right] .
\end{aligned}
$$

Clearly the simplest Weyl covariant object is at the first derivative order:

$$
\hat{\partial}_{t} \Phi+\frac{\delta}{2} \theta \Phi \longrightarrow B^{z+\delta}\left(\hat{\partial}_{t} \Phi+\frac{\delta}{2} \theta \Phi\right) .
$$

This combination was already known in [13] in the context of $z=1$. At the second derivative order we find two such covariant objects:

$$
\begin{aligned}
\hat{\partial}_{t}^{2} \Phi+ & \frac{1}{2}(z+2 \delta) \theta \hat{\partial}_{t} \Phi+\frac{\delta}{4}\left[(z+\delta) \theta^{2}+2 \hat{\partial}_{t} \theta\right] \Phi \\
& \longrightarrow B^{2 z+\delta}\left(\hat{\partial}_{t}^{2} \Phi+\frac{1}{2}(z+2 \delta) \theta \hat{\partial}_{t} \Phi+\frac{\delta}{4}\left[(z+\delta) \theta^{2}+2 \hat{\partial}_{t} \theta\right] \Phi\right) \\
\hat{\nabla}^{i} \hat{\partial}_{i} \Phi & +\frac{2 \delta}{z} \phi^{i} \partial_{i} \Phi-\frac{\delta}{2}\left[\hat{r}-\frac{2 \delta}{z^{2}} \phi^{i} \phi_{i}\right] \Phi \\
& \longrightarrow B^{2+\delta}\left(\hat{\nabla}^{i} \hat{\partial}_{i} \Phi+\frac{2 \delta}{z} \phi^{i} \hat{\partial}_{i} \Phi-\frac{\delta}{2}\left[\hat{r}-\frac{2 \delta}{z^{2}} \phi^{i} \phi_{i}\right] \Phi\right) .
\end{aligned}
$$

Therefore we have found two distinct possibilities for the covariant equations of motion: one with weight $2 z+\delta$, and the other with weight $2+\delta$. We will refer to these as time-like case and space-like case respectively.

These are not yet the most general covariant combinations. At the linear order in $\Phi$, one is free to consider linear combination of (3.17) with $\left(\hat{\gamma}_{j}^{i} \hat{\gamma}_{i}^{j}-\frac{1}{2} \theta^{2}\right) \Phi$ and (3.18) with $\left(\hat{r}+\frac{2}{z} a^{i j} \hat{\nabla}_{i} \phi_{j}\right) \Phi$. At the level of interactions we may consider linear combinations of appropriate powers of $\Phi$ multiplying $\left\{1, \hat{r}+\frac{2}{z} a^{i j} \hat{\nabla}_{i} \phi_{j}, f_{i j} f^{i j}\right\}$ added to (3.17) and $\left\{1, \hat{\gamma}_{j}^{i} \hat{\gamma}_{i}^{j}-\right.$ $\left.\frac{1}{2} \theta^{2}, f_{i j} f^{i j}\right\}$ added to (3.18).

In the interaction terms the powers of $\Phi$ have to be chosen so that the combinations have the right Weyl weights and the potentials are preferably polynomials in $\Phi$. This latter requirement constrains the data $(z, \delta)$. For instance, whenever $\frac{2 z+\delta}{\delta}\left(:=N_{\lambda_{0}}-1\right)$ is a positive integer then one can consider $\Phi^{\frac{2 z+\delta}{\delta}}$ along with (3.17). Similarly in the space-like case one can add $\Phi^{\frac{2+\delta}{\delta}}$ whenever $\frac{2+\delta}{\delta}\left(:=N_{\lambda_{1}}-1\right)$ is a positive integer. Such terms are expected to contribute to the equations of motion when there are monomial potential of the type $\Phi^{N_{\lambda_{0}}}\left(\Phi^{N_{\lambda_{1}}}\right)$ in the action for $\Phi$.

There are two special values of $z$, namely, $z=1$ and $z=2$, that have to be treated more carefully because in these cases we can consider more general covariant combinations.

- $z=1$. We can consider constant linear combinations of the two quantities (3.17) and (3.18) — for in this case the Weyl weights of both of these quantities become 
equal to $2+\delta$. Furthermore precisely in this case the Weyl weights of $\left(\hat{\gamma}_{j}^{i} \hat{\gamma}_{i}^{j}-\frac{1}{2} \theta^{2}\right) \Phi$, $\left(\hat{r}+\frac{2}{z} a^{i j} \hat{\nabla}_{i} \phi_{j}\right) \Phi$ and $f_{i j} f^{i j} \Phi$, (which are all linear in $\Phi$ ) also become $2+\delta$ and so can be added.

- $z=2$. We can consider linear combinations of the first order time-derivative object (3.16) with the space-like derivative object in (3.18) as both their Weyl weights become equal to $2+\delta$.

We can summarise our results so far for various types of Carroll diffeomorphic and Weyl invariant equations of motion for a scalar field $\Phi$ as follows:

- For $z=1$ we can take the covariant equation to be:

$$
\begin{aligned}
& \kappa_{0}\left[\hat{\partial}_{t}^{2} \Phi+\frac{1}{2}(1+2 \delta) \theta \hat{\partial}_{t} \Phi+\frac{\delta}{4}\left[(1+\delta) \theta^{2}+2 \hat{\partial}_{t} \theta\right] \Phi\right] \\
& +\kappa_{1}\left[\hat{\nabla}^{i} \hat{\partial}_{i} \Phi+2 \delta \phi^{i} \hat{\partial}_{i} \Phi-\frac{\delta}{2}\left[\hat{r}-2 \delta \phi^{i} \phi_{i}\right] \Phi\right] \\
& +\left[\sigma_{0}\left(\hat{\gamma}_{j}^{i} \hat{\gamma}_{i}^{j}-\frac{1}{2} \theta^{2}\right)+\sigma_{1}\left(\hat{r}+2 a^{i j} \hat{\nabla}_{i} \phi_{j}\right)+\sigma_{2} f_{i j} f^{i j}\right] \Phi+\lambda \Phi^{\frac{2+\delta}{\delta}}=0 .
\end{aligned}
$$

This equation has five independent real parameters - since $\left(\kappa_{0}, \kappa_{1}, \sigma_{0}, \sigma_{1}, \sigma_{2}, \lambda\right)$ are equivalent to $\alpha\left(\kappa_{0}, \kappa_{1}, \sigma_{0}, \sigma_{1}, \sigma_{2}, \lambda\right)$ for any non-zero real $\alpha$. We may assume that $\left(\kappa_{0}, \kappa_{1}\right) \neq(0,0)$ so that we have a differential equation. Note also that there is no reason to fix $\delta$ at this stage beyond the assumption that $\frac{2+\delta}{\delta}$ is (preferably an odd) positive integer, whenever $\lambda \neq 0$.

- For $z=2$ we can take the covariant equation to be:

$$
\begin{aligned}
\kappa_{3}\left[\hat{\partial}_{t} \Phi+\frac{\delta}{2} \theta \Phi\right] & +\tau_{3}\left[\hat{\nabla}^{i} \hat{\partial}_{i} \Phi+\delta \phi^{i} \hat{\partial}_{i} \Phi-\frac{\delta}{2}\left(\hat{r}-\frac{\delta}{2} \phi^{i} \phi_{i}\right) \Phi\right]+\sigma_{3}\left(\hat{r}+a^{i j} \hat{\nabla}_{i} \phi_{j}\right) \Phi \\
& +\omega_{3}\left(\hat{\gamma}_{j}^{i} \hat{\gamma}_{i}^{j}-\frac{1}{2} \theta^{2}\right) \Phi^{1-\frac{2}{\delta}}+\left(\lambda_{3}+\mu_{3} f_{i j} f^{i j}\right) \Phi^{\frac{2+\delta}{\delta}}=0
\end{aligned}
$$

with Weyl weight $2+\delta$ and (at most) five independent parameters, and/or

$$
\begin{aligned}
\hat{\partial}_{t}^{2} \Phi+(1+\delta) \theta \hat{\partial}_{t} \Phi & +\frac{\delta}{4}\left[(2+\delta) \theta^{2}+2 \hat{\partial}_{t} \theta\right] \Phi+\sigma_{0}\left(\hat{\gamma}_{j}^{i} \hat{\gamma}_{i}^{j}-\frac{1}{2} \theta^{2}\right) \Phi \\
& +\kappa_{0}\left(\hat{r}+a^{i j} \hat{\nabla}_{i} \phi_{j}\right) \Phi^{1+\frac{2}{\delta}}+\left(\lambda_{0}+\mu_{0} f_{i j} f^{i j}\right) \Phi^{\frac{4+\delta}{\delta}}=0
\end{aligned}
$$

with Weyl weight $\delta+4$ and (at most) four parameters.

- For general values of $z$ we have the following two types of Carroll diffeomorphism and Weyl invariant equations of motion

1.

$$
\begin{aligned}
\hat{\partial}_{t}^{2} \Phi+ & \frac{1}{2}(z+2 \delta) \theta \hat{\partial}_{t} \Phi+\frac{\delta}{4}\left[(z+\delta) \theta^{2}+2 \hat{\partial}_{t} \theta\right] \Phi+\sigma_{0}\left(\hat{\gamma}_{j}^{i} \hat{\gamma}_{i}^{j}-\frac{1}{2} \theta^{2}\right) \Phi \\
& +\mu_{0} \Phi^{1+\frac{4}{\delta}(z-1)} f_{i j} f^{i j}+\kappa_{0}\left(\hat{r}+\frac{2}{z} a^{i j} \hat{\nabla}_{i} \phi_{j}\right) \Phi^{1+\frac{2}{\delta}(z-1)}+\lambda_{0} \Phi^{\frac{2 z+\delta}{\delta}}=0
\end{aligned}
$$


2 .

$$
\begin{aligned}
\hat{\nabla}^{i} \hat{\partial}_{i} \Phi+ & \frac{2 \delta}{z} \phi^{i} \hat{\partial}_{i} \Phi-\frac{\delta}{2}\left[\hat{r}-\frac{2 \delta}{z^{2}} \phi^{i} \phi_{i}\right] \Phi+\sigma_{1}\left(\hat{r}+\frac{2}{z} a^{i j} \hat{\nabla}_{i} \phi_{j}\right) \Phi \\
& +\mu_{1} f_{i j} f^{i j} \Phi^{1+\frac{2}{\delta}(z-1)}+\kappa_{1}\left(\hat{\gamma}_{j}^{i} \hat{\gamma}_{i}^{j}-\frac{1}{2} \theta^{2}\right) \Phi^{1+\frac{2}{\delta}(1-z)}+\lambda_{1} \Phi^{\frac{2+\delta}{\delta}}=0,
\end{aligned}
$$

where we assume that the coefficient $\left(\mu_{0}, \kappa_{0}\right)$ and $\left(\mu_{1}, \kappa_{1}\right)$ are non-vanishing only if the powers of $\Phi$ multiplying them are positive integers.

Let us make some comments on the nature of interaction terms in the equations (3.22), (3.23). In the next section we will attempt to construct actions that produce these equations. We will assume that the interaction terms can be obtained from an action with a potential that is a polynomial in $\Phi$ and bounded below. This requires the potential to be a linear combination of positive integer powers of $\Phi$ with appropriate coefficients and the highest power (the degree of the polynomial) being an even number. Further we will assume that the Weyl scaling $\delta$ of $\Phi$ is positive.

- In the time-like case let us denote the potential terms in (3.22) by

$$
\mu_{0} f_{i j} f^{i j} \Phi^{N_{\mu_{0}}-1}+\kappa_{0}\left(\hat{r}+\frac{2}{z} a^{i j} \hat{\nabla}_{i} \phi_{j}\right) \Phi^{N_{\kappa_{0}}-1}+\lambda_{0} \Phi^{N_{\lambda_{0}}-1}
$$

- Then we have $N_{\lambda_{0}}-N_{\kappa_{0}}=\frac{2}{\delta}, N_{\mu_{0}}-N_{\kappa_{0}}=\frac{2}{\delta}(z-1)$ and $N_{\mu_{0}}-N_{\lambda_{0}}=\frac{2}{\delta}(z-2)$. Therefore

1. for $z<1$ we have $N_{\mu_{0}}<N_{\kappa_{0}}<N_{\lambda_{0}}$,

2. for $1<z<2$ we have $N_{\kappa_{0}}<N_{\mu_{0}}<N_{\lambda_{0}}$, and

3. for $z>2$ we have $N_{\kappa_{0}}<N_{\lambda_{0}}<N_{\mu_{0}}$.

Similarly

- for the space-like case let us denote the interaction terms in (3.23) by

$$
\mu_{1} f_{i j} f^{i j} \Phi^{N_{\mu_{1}}-1}+\kappa_{1}\left(\hat{\gamma}_{j}^{i} \hat{\gamma}_{i}^{j}-\frac{1}{2} \theta^{2}\right) \Phi^{N_{\kappa_{1}}-1}+\lambda_{1} \Phi^{N_{\lambda_{1}}-1} .
$$

- Then we have $N_{\lambda_{1}}-N_{\kappa_{1}}=\frac{2 z}{\delta}, N_{\lambda_{1}}-N_{\mu_{1}}=\frac{2}{\delta}(2-z)$ and $N_{\kappa_{1}}-N_{\mu_{1}}=\frac{4}{\delta}(1-z)$. Hence,

1. for $z<0$ we have $N_{\mu_{1}}<N_{\lambda_{1}}<N_{\kappa_{1}}$,

2. for $0<z<1$ we have $N_{\mu_{1}}<N_{\kappa_{1}}<N_{\lambda_{1}}$,

3. for $1<z<2$ we have $N_{\kappa_{1}}<N_{\mu_{1}}<N_{\lambda_{1}}$, and

4. for $z>2$ we have $N_{\kappa_{1}}<N_{\lambda_{1}}<N_{\mu_{1}}$.

Therefore depending on the range of $z$ the degree of the polynomial is different. Demanding that the powers of $\Phi$ are positive integers implies non-trivial constraints on the set of values $(z, \delta)$ can take. As we will see in the next section the existence of actions implies further constraints on these data. So we postpone further discussion of this aspect for later. 


\subsection{Constructing actions}

Now that we have derived the most general diffeomorphic and Weyl covariant equations of motion for a single real ${ }^{1}$ scalar field $\Phi$ in the background of generic Carroll geometry, we would like to turn to constructing the corresponding actions next. We should seek actions that produce each of the equations ((3.19), (3.20), (3.21), (3.22), (3.23)) as their Euler-Lagrange equations for the scalar field $\Phi$.

Note that our equations of motion in the absence of interactions are linear in $\Phi$ and have up to two derivatives on $\Phi$. If we are to be able to derive them from some actions then they should be quadratic in $\Phi$ and should contain up to two derivatives. Therefore let us again start by listing all such invariants - now counting derivatives both on the background geometric quantities $\left(a_{i j}, b_{i}, \omega\right)$ and on $\Phi$.

$$
\begin{aligned}
& \theta \Phi^{2}, \quad \Phi \hat{\partial}_{t} \Phi, \\
& \hat{\gamma}_{j}^{i} \hat{\gamma}_{i}^{j} \Phi^{2}, \quad \theta^{2} \Phi^{2}, \quad \theta \Phi \hat{\partial}_{t} \Phi, \quad \hat{\partial}_{t} \theta \Phi^{2}, \quad\left(\hat{\partial}_{t} \Phi\right)^{2}, \quad \Phi \hat{\partial}_{t}^{2} \Phi, \\
& \hat{r} \Phi^{2}, \quad \hat{\nabla}_{i} \phi^{i} \Phi^{2}, \quad \phi^{i} \phi_{i} \Phi^{2}, \quad f_{i j} f^{i j} \Phi^{2}, \quad \Phi \phi^{i} \hat{\partial}_{i} \Phi, \quad \hat{\partial}_{i} \Phi \hat{\partial}_{i} \Phi, \quad \Phi \hat{\nabla}^{i} \hat{\partial}_{i} \Phi .
\end{aligned}
$$

We have already listed in (3.15) all the transformations required under the Carroll Weyl transformations and we simply have to find the combinations of quantities in (3.26) that transform homogeneously.

After a straightforward analysis using the results of the previous subsection we find the following combinations up to quadratic order in $\Phi$ :

1. At first order in time-derivatives we have the unique combination with weight $z+2 \delta$

$$
\Phi\left(\hat{\partial}_{t} \Phi+\frac{\delta}{2} \theta \Phi\right)
$$

2. At second order in time-derivatives we find three covariant combinations with weight $2(z+\delta)$.

$$
\begin{gathered}
\left(\hat{\partial}_{t} \Phi+\frac{\delta}{2} \theta \Phi\right)^{2}, \quad\left(\hat{\gamma}_{j}^{i} \hat{\gamma}_{i}^{j}-\frac{1}{2} \theta^{2}\right) \Phi^{2} \\
\left(\hat{\partial}_{t} \theta+\frac{z}{2} \hat{\gamma}_{j}^{i} \hat{\gamma}_{i}^{j}\right) \Phi^{2}+\frac{2}{\delta} \Phi \hat{\partial}_{t}^{2} \Phi-\frac{2 \delta+z}{\delta^{2}}\left(\hat{\partial}_{t} \Phi\right)^{2}
\end{gathered}
$$

We will refer to these as time-like combinations.

3. At second order in space-derivatives we find three combinations with Weyl weight $2(1+\delta):$

$$
\begin{gathered}
\left(\hat{\partial}_{i} \Phi+\frac{\delta}{z} \phi_{i} \Phi\right)\left(\hat{\partial}^{i} \Phi+\frac{\delta}{z} \phi^{i} \Phi\right), \quad\left(\hat{r}+\frac{2}{z} \hat{\nabla}_{i} \phi^{i}\right) \Phi^{2}, \\
\Phi \hat{\nabla}_{i} \hat{\partial}^{i} \Phi+2 \frac{\delta}{z} \Phi \phi^{i} \hat{\partial}_{i} \Phi+\frac{\delta}{z}\left(\hat{\nabla}_{i} \phi^{i}+\frac{\delta}{z} \phi_{i} \phi^{i}\right) \Phi^{2}
\end{gathered}
$$

We refer to these as space-like combinations.

\footnotetext{
${ }^{1}$ For complex $\Phi$ one simply has to replace the potentials to be appropriate real combinations, such as $|\Phi|^{2 n}$ etc.
} 
At higher orders in $\Phi$ we can consider potentials with coefficients from any of

$$
1, \quad f_{i j} f^{i j}, \quad \hat{r}+\frac{2}{z} \hat{\nabla}_{i} \phi^{i}, \quad \hat{\gamma}_{j}^{i} \hat{\gamma}_{i}^{j}-\frac{1}{2} \theta^{2}
$$

Now any candidate action has to be an integral over the coordinates $(t, \mathbf{x})$ of our Carroll manifold:

$$
S=\int d t d^{2} \mathbf{x} \mathcal{L}
$$

For the action to be invariant under diffeomorphisms the Lagrangian density should transform as $\mathcal{L} \rightarrow \mathcal{L}^{\prime}$, such that

$$
\int d t d^{2} \mathbf{x} \mathcal{L}=\int d t^{\prime} d^{2} \mathbf{x}^{\prime} \mathcal{L}^{\prime}
$$

From (3.1) we have $d t^{\prime} d^{2} \mathbf{x}^{\prime}=J \operatorname{det} J_{j}^{i} d t d^{2} \mathbf{x}$. So the Lagrangian density $\mathcal{L}$ has to transform as $\mathcal{L} \rightarrow \mathcal{L}^{\prime}=J^{-1} \operatorname{det}\left(\left(J^{-1}\right)_{j}^{i}\right) \mathcal{L}-i$.e, as a scalar density of weight 3 , equal to the dimension of the manifold - under the relevant Carroll diffeomorphisms. The combinations we listed above in (3.27)-(3.31) are all scalars under Carroll diffoemorphisms, even though they have non-trivial weights under Carroll Weyl transformations. So to make them densities of suitable weights we need to multiply them by $\omega \sqrt{a}$ as this is the only combination of the Carroll geometry without derivatives and transforms as desired:

$$
\omega \sqrt{a} \rightarrow J^{-1} \operatorname{det}\left(\left(J^{-1}\right)_{j}^{i}\right) \omega \sqrt{a}
$$

where $a$ is the determinant of the metric $a_{i j}$ on the base space. For instance

$$
S=\int d t d^{2} \mathbf{x} \omega \sqrt{a}\left[\hat{\partial}_{i} \Phi \hat{\partial}^{i} \Phi+2 \frac{\delta}{z} \Phi \phi^{i} \hat{\partial}_{i} \Phi+\frac{\delta^{2}}{z^{2}} \phi_{i} \phi^{i} \Phi^{2}\right],
$$

is a good action for a Carroll diffeomorphism invariant theory. However, for it to be also a Weyl invariant theory the Lagrangian density $\mathcal{L}$ has to be invariant by itself (up to totaldivergence terms) under (3.11). Let us check this for (3.36): the measure transforms as $\omega \sqrt{a} \rightarrow B^{-2-z} \omega \sqrt{a}$ and the quantity in square-brackets transforms as $[\cdots] \rightarrow B^{2+2 \delta}[\cdots]$ under the Weyl transformations (3.11). So demanding that the Lagrangian density $\mathcal{L}$ in (3.36) is Weyl invariant requires

$$
2+2 \delta=2+z \Longrightarrow \delta=\frac{z}{2} .
$$

This conclusion is valid for all actions constructed using the space-like combinations with Weyl weight $2+2 \delta$. Also $\delta>0$ requires $z>0$.

Similarly if we use any of the Carroll diffeomorphism invariant and Carroll Weyl covariant time-like combinations in (3.28) we need to fix the weight $\delta$ of the scalar such that:

$$
2+z=2 z+2 \delta \Longrightarrow \delta=1-\frac{z}{2} .
$$

In this case for $\delta>0$ we need to have $z<2$.

Note that this is unlike the conformally coupled scalar in the background of a (pseudo) Riemann manifold where the Weyl weight of the scalar is fixed at the level of equation of 
motion itself (as reviewed in section 2), whereas for the scalar in Carroll geometry it is fixed (even for a free scalar) at the level of the existence of an action.

When we include interactions, we have to continue to impose the constraints (3.38) in time-like case and (3.37) in the space-like case. So we have the potential:

- in the time-like case is $(\omega \sqrt{a}$ times $)$

$$
\frac{1}{N_{\mu_{0}}} \mu_{0} f_{i j} f^{i j} \Phi^{N_{\mu_{0}}}+\frac{1}{N_{\kappa_{0}}} \kappa_{0}\left(\hat{r}+\frac{2}{z} a^{i j} \hat{\nabla}_{i} \phi_{j}\right) \Phi^{N_{\kappa_{0}}}+\frac{1}{N_{\lambda_{0}}} \lambda_{0} \Phi^{N_{\lambda_{0}}}
$$

with $N_{\mu_{0}}=\frac{4}{\delta}-6, N_{\kappa_{0}}=\frac{2}{\delta}-2$ and $N_{\lambda_{0}}=\frac{4}{\delta}-2$. We further assume that $N_{\mu_{0}}, N_{\kappa_{0}}, N_{\lambda_{0}} \geq 2$. For $N_{\mu_{0}} \geq 2$ and $N_{\kappa_{0}} \geq 2$ we need $\delta \leq \frac{1}{2}$ and $N_{\lambda_{0}} \geq 2$ requires $\delta \leq 1$. Thus we can have all three terms in the potential only when $0 \leq \delta \leq \frac{1}{2}$ with $N_{\lambda_{0}}$ being the largest power. When $\frac{1}{2}<\delta \leq 1$ we can only have $\lambda_{0}$ non-zero and if $\delta>1$ no potential is possible.

- In the space-like case it is $(\omega \sqrt{a}$ times)

$$
\frac{1}{N_{\mu_{1}}} \mu_{1} f_{i j} f^{i j} \Phi^{N_{\mu_{1}}}+\frac{1}{N_{\kappa_{1}}} \kappa_{1}\left(\hat{\gamma}_{j}^{i} \hat{\gamma}_{i}^{j}-\frac{1}{2} \theta^{2}\right) \Phi^{N_{\kappa_{1}}}+\frac{1}{N_{\lambda_{1}}} \lambda_{1} \Phi^{N_{\lambda_{1}}}
$$

with $N_{\mu_{1}}=6-\frac{2}{\delta}, N_{\kappa_{1}}=\frac{2}{\delta}-2$ and $N_{\lambda_{1}}=\frac{2}{\delta}+2$. We again assume that $N_{\mu_{1}}, N_{\kappa_{1}}, N_{\lambda_{1}} \geq$ 2. For $N_{\mu_{1}} \geq 2$ requires $\delta \geq \frac{1}{2}$, for $N_{\kappa_{1}} \geq 2$ requires $\delta \leq \frac{1}{2}$, and for $N_{\lambda_{1}} \geq 2$ requires $\delta \geq 0$. So when we take $0 \leq \delta<\frac{1}{2}$ (which means $0 \leq z<1$ ) we need to set $\mu_{1}=0$, and we have $N_{\lambda_{1}}$ as the largest power. When $\frac{1}{2}<\delta \leq 1$ we cannot have $\kappa_{1}$ non-zero and the highest power of the potential is again given by $N_{\lambda_{1}}$. When $\delta>1$ we still have $\kappa_{1}=0$ and the largest power of $\Phi$ in the potential will be $N_{\mu_{1}}$. Only when $\delta=\frac{1}{2}$ all three terms are possible with $N_{\lambda_{1}}=6$.

To summarise in both time-like and space-like cases the different types of interactions are possible depending on the values of $\delta$ (determined in terms of $z$ via (3.38), (3.37)). When $0 \leq \delta \leq 1$ the dominant power in the potential comes from the monomial $\lambda_{0} \Phi^{N_{\lambda_{0}}}\left(\lambda_{1} \Phi^{N_{\lambda_{1}}}\right)$ in the time-like (space-like) case. When $\delta>1$ no potential is possible in the time-like case and in the space-like case the dominant power is given by $N_{\mu_{1}}$. Finally we have to impose that the dominant power of the potential is even.

- In the time-like case, whenever the potential is allowed the degree of the potential is $N_{\lambda_{0}}$. For the potential to be bounded below we require that $N_{\lambda_{0}}$ is even, i.e., $N_{\lambda_{0}}=2 n_{t}$ for some $n_{t} \geq 1$. Thus the data $(z, \delta)$ are restricted to:

$$
\delta=1-\frac{z}{2}=\frac{z}{n_{t}-1} \Longrightarrow z=2 \frac{n_{t}-1}{n_{t}+1} \quad \delta=\frac{2}{n_{t}+1}, \quad n_{t} \geq 1
$$

In this case the range of $z$ is between 0 (when $n_{t}=1$ ) and 2 (when $n_{t} \rightarrow \infty$ ). The case of $n_{t}=3$ gives $z=1$ with $\delta=1 / 2$, and this is what one obtains by taking the ultra-relativistic limit of conformally coupled scalar in 3 dimensions - as we show in section 4 . 
- In the space-like case when $0<\delta \leq 1$ we take $N_{\lambda_{1}}=2 n_{s}$ for some $n_{s} \geq 2$ (since when $0<\delta \leq 1$ we have $N_{\lambda_{1}} \geq 4$ ). Then we have

$$
\delta=\frac{z}{2}=\frac{1}{n_{s}-1} \Longrightarrow z=\frac{2}{n_{s}-1} \quad \delta=\frac{1}{n_{s}-1}, \quad n_{s} \geq 2 .
$$

This is the set of values assumed for $z$ by Duval et al. [5], where they denoted $k=n_{s}-1$ with $k \geq 1$. In this case the range of $z$ is between zero (when $n_{s} \rightarrow \infty$ ) and 2 (when $n_{s}=2$ ) with the corresponding values of $\delta$ ranging between 0 and 1 . Again the special value $z=1$ gives $\delta=1 / 2$.

When $\delta>1$ we need to take $N_{\mu_{1}}=2 n_{\mu}$ for some $n_{\mu} \geq 3$ since $N_{\mu_{1}}=6-\frac{2}{\delta}$. At the same time $N_{\mu_{1}}$ is bounded above by 6 and so the only non-trivial possibility is $n_{\mu}=3$. But when $n_{\mu}=3$ both $z$ and $\delta$ are infinite which we do not consider.

So we conclude that the existence of (i) bounded and polynomial potentials and (ii) invariant actions implies discrete and specific rational values for both $z$ and $\delta$.

Actions for equations. We now propose actions which produce (3.19)-(3.23) as their equations of motion.

- For the time-like case with $z=2(1-\delta)$ our action is

$$
S_{t}=\int d t d^{2} \mathbf{x} \omega \sqrt{a}\left[\alpha_{1}\left(\hat{\partial}_{t} \Phi+\frac{\delta}{2} \theta \Phi\right)^{2}+\beta_{1}\left(\hat{\gamma}_{j}^{i} \hat{\gamma}_{i}^{j}-\frac{1}{2} \theta^{2}\right) \Phi^{2}+\lambda_{1} \Phi^{2+\frac{2 z}{\delta}}\right],
$$

which gives the equation of motion for $\Phi$ to be:

$$
\begin{aligned}
-2 & \alpha_{1}\left[\hat{\partial}_{t}^{2} \Phi+\theta \hat{\partial}_{t} \Phi-\frac{1}{4} \delta(\delta-2) \theta^{2} \Phi+\frac{\delta}{2} \hat{\partial}_{t} \theta \Phi\right] \\
& +2 \beta_{1}\left(\hat{\gamma}_{j}^{i} \hat{\gamma}_{i}^{j}-\frac{1}{2} \theta^{2}\right) \Phi+2 \lambda_{1}\left(\frac{2}{\delta}-1\right) \Phi^{\frac{4}{\delta}-3}=0
\end{aligned}
$$

- For the space-like case with $z=2 \delta$ our action is

$$
\begin{aligned}
S_{s}= & \int d t d^{2} \mathbf{x} \omega \sqrt{a} \\
& \times\left[\alpha_{2}\left(\hat{\partial}_{i} \Phi+\frac{\delta}{z} \phi_{i} \Phi\right)\left(\hat{\partial}^{i} \Phi+\frac{\delta}{z} \phi^{i} \Phi\right)+\beta_{2}\left(\hat{r}+\frac{2}{z} \hat{\nabla}_{i} \phi^{i}\right) \Phi^{2}+\lambda_{2} \Phi^{2+\frac{2}{\delta}}\right],
\end{aligned}
$$

which gives rise to

$$
\begin{aligned}
& -2 \alpha_{2}\left[\hat{\square} \Phi+\phi^{i} \hat{\nabla}_{i} \Phi+\frac{1}{4} \phi^{i} \phi_{i} \Phi+\frac{1}{2} \hat{\nabla}_{i} \phi^{i} \Phi\right] \\
& +2 \beta_{2}\left(\hat{r}+\frac{2}{z} \hat{\nabla}_{i} \phi^{i}\right) \Phi+2 \lambda_{2}\left(1+\frac{1}{\delta}\right) \Phi^{1+\frac{2}{\delta}}=0 .
\end{aligned}
$$

It is easy to see that these equations are the same as (3.22)-(3.23) with appropriate identifications of the coefficients. $^{2}$

\footnotetext{
${ }^{2}$ One can easily incorporate other interaction terms into the actions to produce potential terms in $(3.22),(3.23)$ with non-constant coefficients as well whenever they are allowed.
} 
For the special case $z=1$ we can consider linear combinations of (3.43), (3.45) with (3.31), which can be seen to generate equations of the form (3.19).

The other special value of $z$, namely $z=2$ needs some more attention. In this case we either have to consider a complex $\Phi$ or two real fields $\Phi_{1}$ and $\Phi_{2}$. To see this we first note that in this case we expect an action to reproduce an equation of motion of the type (3.20) that includes (whenever $\kappa_{3} \neq 0$ ) first-order time-derivatives. Using a single real scalar a candidate action that could have produced a first-order time-derivative term is $\omega \sqrt{a} \Phi\left(\hat{\partial}_{t} \Phi+\frac{\delta}{2} \theta \Phi\right)$. But such an action does not lead to non-trivial equation of motion in $z=2$ case (which in turn requires $\delta=1)$ since $\omega \sqrt{a} \Phi\left(\hat{\partial}_{t} \Phi+\frac{1}{2} \theta \Phi\right)=\partial_{t}\left(\frac{1}{2} \sqrt{a} \Phi^{2}\right)$ is a total derivative. A simple way to overcome this is to consider $\omega \sqrt{a} \Phi^{\star}\left(\hat{\partial}_{t} \Phi+\frac{\delta}{2} \theta \Phi\right)$ for a complex $\Phi$ with $\Phi^{\star}$ being its complex conjugate.

\section{A Carroll CFT from a conformally coupled scalar}

Now we turn to show that some special cases of the equations of motion and their corresponding actions we derived in the last section arise in the ultra-relativistic limit of the conformally coupled scalar reviewed in section 2 .

Our starting point is the diffeomorphic and Weyl invariant scalar field theory in $2+1$ dimensional spacetime. The background metric is taken in the so-called Randers-Papapetrou form (see for instance [13]), with the line element:

$$
d s^{2}=g_{\mu \nu} d x^{\mu} d x^{\nu}=-c^{2} \omega^{2}\left(d t-\omega^{-1} b_{i} d x^{i}\right)^{2}+a_{i j} d x^{i} d x^{j}
$$

where $x^{\mu}=(t, \mathbf{x})$. This geometry in the $c \rightarrow 0$ limit is expected to produce a Carroll geometry. Moreover the subset of all diffeomorphisms that leave this metric form-invariant are precisely the Carroll diffeomorphisms (3.1) and the quantities $\left\{a_{i j}, b_{i}, \omega\right\}$ transform under (3.1) as in (3.4).

It can be seen that the Ricci scalar of this geometry is

$$
R=c^{-2}\left(\theta^{2}+\hat{\gamma}_{j}^{i} \hat{\gamma}_{i}^{j}+2 \hat{\partial}_{t} \theta\right)+\left(\hat{r}-2 \hat{\nabla}_{i} \phi^{i}-2 \phi_{i} \phi^{i}\right)+\frac{c^{2}}{4} f_{i j} f^{i j} .
$$

Consider the Weyl invariant Klein-Gordon scalar field equation on a general background in three dimensions:

$$
g^{a b} \nabla_{a} \nabla_{b} \Phi-\frac{1}{8} R \Phi=0
$$

Using Randers-Papapetrou metric ansatz (4.1) one can reduce this equation to a polynomial in $c$. This equation admits an expansion in terms of the combinations in (3.19)-(3.23) with $(z, \delta)=\left(1, \frac{1}{2}\right)$. In particular:

$$
\begin{aligned}
\hat{\square} \Phi-\frac{1}{8} R \Phi= & -\frac{1}{c^{2}}\left[\hat{\partial}_{t}^{2} \Phi+\theta \hat{\partial}_{t} \Phi+\frac{1}{16}\left(3 \theta^{2}+4 \hat{\partial}_{t} \theta\right) \Phi\right]-\frac{1}{8 c^{2}}\left(\hat{\gamma}_{j}^{i} \hat{\gamma}_{i}^{j}-\frac{1}{2} \theta^{2}\right) \\
& +\left[\hat{\square} \Phi+\phi^{i} \hat{\nabla}_{i} \Phi-\frac{1}{4}\left(\hat{r}-\phi^{i} \phi_{i}\right) \Phi\right]+\frac{1}{8}\left(\hat{r}+2 \hat{\nabla}_{i} \phi^{i}\right) \Phi \\
& -\frac{c^{2}}{32} f_{i j} f^{i j} \Phi .
\end{aligned}
$$


Notice that if we had set any of these terms at any given order in powers of $c$ to zero, it would have given us a Carroll diffeomorphic and Weyl covariant equation. We do not have to just restrict to the lowest order term (the leading term in the ultra-relativistic limit - as was done in the previous works [3-5, 11-17]). However, if we took the linear combination exactly as in (4.4) we can re-package the equation into a fully diffeomorphic and Weyl covariant equation (4.3) in the pseudo-Riemannian space with metric (4.1).

Let us now turn to the action in this special case. For this we start with the Lagrangian density of the conformally coupled scalar to the background Randers-Papapetrou metric (4.1) and again expand it in powers of $c$. This gives us:

$$
\begin{aligned}
S= & \frac{1}{c} \int d t d^{2} \mathbf{x} \omega \sqrt{a}\left[\left(\hat{\partial}_{t} \Phi\right)^{2}-\frac{1}{8}\left(\theta^{2}+\hat{\gamma}_{j}^{i} \hat{\gamma}_{i}^{j}+2 \hat{\partial}_{t} \theta\right) \Phi^{2}\right] \\
& -c \int d t d^{2} \mathbf{x} \omega \sqrt{a}\left[\hat{\nabla}_{i} \Phi \hat{\nabla}^{i} \Phi+\frac{1}{8}\left(\hat{r}-2 \hat{\nabla}_{i} \phi^{i}-2 \phi_{i} \phi^{i}\right) \Phi^{2}\right] \\
& -\frac{c^{3}}{32} \int d t d^{2} \mathbf{x} \omega \sqrt{a} f_{i j} f^{i j} \Phi^{2} .
\end{aligned}
$$

If we put $\alpha_{1}=\frac{1}{c}$ and $\beta_{1}=-\frac{1}{8 c}\left(\right.$ with $\left.\lambda_{1}=0\right)$ and use

$$
\partial_{t}\left(\sqrt{a} \theta \Phi^{2}\right)=\omega \sqrt{a}\left[2 \theta \Phi \hat{\partial}_{t} \Phi+\left(\theta^{2}+\hat{\partial}_{t} \theta\right) \Phi^{2}\right]
$$

we see that the $\mathcal{O}(1 / c)$ term of this action is a special case of $(3.43)$ with $(z, \delta)=\left(1, \frac{1}{2}\right)$ up to a total derivative. Similarly noticing that

$$
\partial_{i}\left[\omega \sqrt{a} \phi^{i} \Phi^{2}\right]+\partial_{t}\left[\sqrt{a} b_{i} \phi^{i} \Phi^{2}\right]=\omega \sqrt{a}\left[2 \Phi \phi^{i} \hat{\partial}_{i} \Phi+\left(\phi^{i} \phi_{i}+\hat{\nabla}_{i} \phi^{i}\right) \Phi^{2}\right]
$$

and setting $\alpha_{2}=1, \beta_{2}=\frac{1}{8}$ we can see that the $\mathcal{O}(c)$ term in this action is again a special case of (3.45). Finally the order $c^{3}$ term can be added for free again in this case of $z=1$ and $\delta=1 / 2$ as before.

\section{$5 \quad$ Gauge fixing and residual symmetries}

So far we have been using both Carroll diffeomorphisms and Carroll Weyl symmetries to constrain our theories. However, our main interest is in getting down to Carrollian CFTs. For this we need to fix the background spacetime geometry — as much as possible using the local symmetries. In the standard construction of CFTs in the background of a (pseudo) Riemannian manifold one takes a fixed background metric (or a representative in its conformal class). Then the local symmetries (in $(d+1)$-dimensional case) can be used to fix $d+2$ components of the metric completely. This allows one to gauge fix any metric to a specific member of its conformal class. Furthermore if one wants to get a conformal field theory with symmetry algebra $s o(2, d+1)$ then the background should be conformally flat. This condition requires that the metric $g_{\mu \nu}$ should have vanishing Weyl tensor: $W_{\mu \nu \sigma \lambda}=0$ (or the Cotton tensor $C_{\mu \nu}=0$ in $d=2$ case).

Turning now to the Carroll case we again have in a generic geometry, specified by $\left(a_{i j}, b_{i}, \omega\right)$, as many as $\frac{1}{2}(d+1)(d+2)$ components which are all arbitrary functions of $(t, \mathbf{x})$. 
But the local symmetries have two functions $\left(t^{\prime}(t, \mathbf{x}), B(t, \mathbf{x})\right)$ of both space and time and $d$ functions $\left(x^{i}(\mathbf{x})\right)$ of space alone.

We are looking for 3-dimensional Carrollian CFTs with symmetry algebra $\mathfrak{c c a}_{3}^{(z)}$ that contains the conformal algebra of the two dimensional base space $s o(1,3)$ - so we would like to be able to completely gauge fix $a_{i j}$ to some fixed time-independent one. One possibility is that we restrict to Carroll geometries where $a_{i j}$ is of the form:

$$
a_{i j}(t, \mathbf{x})=e^{\chi(t, \mathbf{x})} a_{i j}^{(0)}(\mathbf{x}), \quad \operatorname{det} a_{i j}^{(0)}(\mathbf{x})=\text { fixed. }
$$

In other words the metric $a_{i j}$ is conformally time-independent. Then we will have two components in $a_{i j}^{(0)}(\mathbf{x})$ which can be gauge fixed completely using the spatial diffeomorphisms alone. Then we can use the temporal diffeomorphism and the Weyl symmetry to gauge fix $\chi(t, \mathbf{x})=0$ and $\omega(t, \mathbf{x})=1$. It turns out (as shown later on in this section) that this will be sufficient to ensure that the residual symmetry algebra to be $\mathfrak{c c a}_{3}^{(z)}$. Also the covariant condition analogous to vanishing Weyl or Cotton tensors in the pseudo-Riemannian case, is $\hat{\gamma}_{i j}-\frac{1}{2} \theta a_{i j}=0$.

Some examples of Carroll spacetimes include: Flat Carroll spacetime [3-5, 11] given by

$$
a_{i j}=\delta_{i j}, \quad b_{i}=b_{i}^{(0)}, \quad \omega=1
$$

where $b_{i}^{(0)}$ are constants. For this we have $\theta=\phi_{i}=\hat{\gamma}_{j}^{i}=f_{i j}=\hat{\gamma}_{j k}^{i}=0$. The timelike action (3.43) is what people obtained by ultra-relativistic limit of the Klein-Gordon scalar in 3-dimensional Minkowski spacetime $M_{2+1}$. The space-like action (3.45) becomes, for $z=2 \delta$

$$
\int d t d^{2} \mathbf{x}\left[\hat{\partial}_{i} \Phi \hat{\partial}_{i} \Phi+\lambda \Phi^{2+\frac{2}{\delta}}\right] .
$$

More general Carroll manifolds that include some of the interesting Carroll spacetimes, such as, null infinities, black hole horizons etc, and their conformal symmetries are discussed in detail in [11]. To study the symmetries of the gauge fixed actions one takes the vector field that generates Carroll diffeomorphism to be of the form:

$$
\begin{aligned}
\xi & =f(t, \mathbf{x}) \hat{\partial}_{t}+\xi^{i}(\mathbf{x}) \hat{\partial}_{i} \\
& =\omega^{-1}\left(f+\xi^{i} b_{i}\right) \partial_{t}+\xi^{i} \partial_{i}
\end{aligned}
$$

Under the infinitesimal coordinate transformation

$$
t^{\prime}=t+\omega^{-1}\left(f+\xi^{i} b_{i}\right)+\cdots, \quad x^{i}=x^{i}+\xi^{i}+\cdots
$$

the background data $\left(a_{i j}, b_{i}, \omega\right)$ and of matter field $\Phi$ transform as:

$$
\begin{aligned}
\delta_{\xi} a_{i j} & =-2 f \hat{\gamma}_{i j}-\left(\hat{\nabla}_{i} \xi_{j}+\hat{\nabla}_{j} \xi_{i}\right), \\
\omega^{-1} \delta_{\xi} \omega & =-\hat{\partial}_{t} f-\phi_{i} \xi^{i}, \\
\delta_{\xi} b_{i} & =-b_{i}\left(\phi_{j} \xi^{j}+\hat{\partial}_{t} f\right)+f_{i j} \xi^{j}+\left(\hat{\partial}_{i}-\phi_{i}\right) f, \\
\delta_{\xi} \Phi & =-\left(f \hat{\partial}_{t} \Phi+\xi^{i} \hat{\partial}_{i} \Phi\right) .
\end{aligned}
$$


Under the infinitesimal Weyl transformations with $B=e^{\sigma}$, we have

$$
\begin{aligned}
& \delta_{\sigma} a_{i j}=-2 \sigma a_{i j}, \quad \delta_{\sigma} \omega=-z \sigma \omega, \\
& \delta_{\sigma} b_{i}=-z \sigma b_{i}, \quad \delta_{\sigma} \Phi=\delta \sigma \Phi .
\end{aligned}
$$

One first demands that the metric on the base $a_{i j}$ is invariant under the combined action (5.6), (5.7):

$$
\left(\delta_{\xi}+\delta_{\sigma}\right) a_{i j}=0
$$

and this leads to

$$
2 f\left[\hat{\gamma}_{i j}-\frac{1}{2} \theta a_{i j}\right]+\hat{\nabla}_{i} \xi_{j}+\hat{\nabla}_{j} \xi_{i}-\hat{\nabla}_{k} \xi^{k} a_{i j}=0, \quad \sigma=-\frac{1}{2}\left(f \theta+\hat{\nabla}_{i} \xi^{i}\right) .
$$

Now following [11] we will also choose to impose that the traceless symmetric tensor $\zeta_{i j}=$ $\hat{\gamma}_{i j}-\frac{1}{2} \theta a_{i j}$ (referred to as the Carroll shear) vanishes. This condition can be solved for and it implies (5.1) and hence $a_{i j}$ is conformally time independent. Next we choose to impose $\left(\delta_{\xi}+\delta_{\sigma}\right) \omega=0$ and this leads to

$$
\begin{aligned}
\left(\hat{\partial}_{t}-\frac{z}{2} \theta\right) f-\frac{z}{2}\left(\hat{\nabla}_{i}-\frac{2}{z} \phi_{i}\right) \xi^{i} & =0, \\
\text { along with } \quad \hat{\nabla}_{i} \xi_{j}+\hat{\nabla}_{j} \xi_{i}-\hat{\nabla}_{k} \xi^{k} a_{i j} & =0 .
\end{aligned}
$$

Finally noticing that these equations are Carroll Weyl invariant one chooses $a_{i j}$ to be completely time independent fixed metric. This implies that $\theta=0$ and the Carroll LeviCivita connection $\hat{\gamma}_{j k}^{i}$ reduces to the Christoffel connection for (now time independent) $a_{i j}$. One also chooses a fixed background value for $\omega$ (say, $\omega=1$ ) without loss of generality. Then the residual symmetries have to satisfy

$$
\begin{aligned}
\nabla_{i} \xi_{j}+\nabla_{j} \xi_{i}-\nabla_{k} \xi^{k} a_{i j} & =0, \\
\partial_{t} f-\frac{z}{2}\left(\nabla_{i}-\frac{2}{z} \phi_{i}\right) \xi^{i} & =0 .
\end{aligned}
$$

One can integrate these equations completely and the result is given in terms of $\left\{T(\mathbf{x}), Y^{i}(\mathbf{x}\}\right.$ where $\xi^{i}=Y^{i}(\mathbf{x})$ are the conformal Killing vectors on $a_{i j}$ and $T(\mathbf{x})$ is arbitrary:

$$
\begin{aligned}
f(t, \mathbf{x}) & =T(\mathbf{x})+\frac{z}{2} \int^{t} d t^{\prime}\left[\nabla_{i} Y^{i}(\mathbf{x})-\frac{2}{z} \phi_{i} Y^{i}(\mathbf{x})\right] \\
& =T(\mathbf{x})+\frac{z}{2} t \nabla_{i} Y^{i}(\mathbf{x})-b_{i}(t, \mathbf{x}) Y^{i}(\mathbf{x})
\end{aligned}
$$

where we have set $\omega(t, \mathbf{x})=1$ and $\phi_{i}=\partial_{t} b_{i}$. So the Carrollian conformal Killing vector is

$$
\begin{aligned}
\xi & =\left(f+\xi^{i} b_{i}\right) \partial_{t}+\xi^{i} \partial_{i} \\
& =\left[T(\mathbf{x})+\frac{z}{2} t \nabla_{i} Y^{i}(\mathbf{x})\right] \partial_{t}+Y^{i}(\mathbf{x}) \partial_{i}
\end{aligned}
$$

It is argued in [11] that the algebra of the Carrollian CKV do not depend on the choice of $\omega$ either. The fact that the $b_{i}$ dependence canceled out in the final answer (5.13) 
is also general enough that we do not need to fix $b_{i}$ to define the residual symmetries. Therefore the residual fields $b_{i}(t, \mathbf{x})$ along with $\Phi$ transform under the Carrollian conformal transformations as:

$$
\begin{aligned}
& \delta b_{i}=-b_{i}\left(\phi_{j} \xi^{j}+\hat{\partial}_{t} f\right)+f_{i j} \xi^{j}+\left(\hat{\partial}_{i}-\phi_{i}\right) f-z \sigma b_{i}, \\
& \delta \Phi=-\left(f \hat{\partial}_{t} \Phi+\xi^{i} \hat{\partial}_{i} \Phi\right)+\delta \sigma \Phi,
\end{aligned}
$$

where we have to use $a_{i j}$ to be fixed and time independent (for instance that of a round $S^{2}$ ), $\omega=1$ (and hence $\left.\phi_{i}=\partial_{t} b_{i}\right), \xi^{i}=Y^{i}(\mathbf{x})$ and $f(t, \mathbf{x})$ as in (5.12). These form the symmetries of our theories by construction when we gauge fix as above. This means that our $\mathfrak{c c a}_{3}^{(z)}$ symmetric scalar field theories, after gauge fixing the Carroll diffeomorphisms and Weyl symmetries would have the matter field $\Phi(t, \mathbf{x})$ along with the geometric fields $b_{i}(t, \mathbf{x})$ dynamical. Then the transformations (5.14) are their symmetries, and can be used to study the conserved currents and charges etc. of our theories both at classical level and beyond.

There may be other interesting possibilities to gauge fix the local symmetries with apparently different residual symmetry algebras - we discuss one such gauge now. Let us consider restricting the background Carroll geometric data $\left(a_{i j}, b_{i}, \omega\right)$ by imposing $\phi_{i}=0$. This condition can be solved as follows:

$$
\frac{1}{\omega}\left(\partial_{i} \omega+\partial_{t} b_{i}\right)=0 \Longrightarrow b_{i}(t, \mathbf{x})=b_{i}^{(0)}(\mathbf{x})-\partial_{i} \int^{t} d t^{\prime} \omega\left(t^{\prime}, \mathbf{x}\right)
$$

If we further gauge fix $\omega=1$ as in the previous gauge choice we conclude that $\phi_{i}=0$ implies that $b_{i}$ is independent of time. So we can use the spatial Carroll diffeomorphisms to gauge fix $b_{i}=0 .{ }^{3}$ Finally we can use the Weyl symmetry to fix the determinant of $a_{i j}$, say $a=1$, which in turn will imply $\theta=0$. So in this gauge we have $\phi_{i}=\theta=b_{i}=f_{i j}=0$. Using $\left(\delta_{\xi}+\delta_{\sigma}\right) b_{i}=0$ leads to $\partial_{i} f=0$. From $\left(\delta_{\xi}+\delta_{\sigma}\right) \omega=0$ we find $\sigma=-\frac{1}{z} \partial_{t} f(t)$. This gauge leaves $a_{i j}(t, \mathbf{x})$ to fluctuate subject to the condition $a=1$ along with $\Phi(t, \mathbf{x})$. The condition $a=1$ also implies $\sigma=-\frac{1}{2} \nabla_{i} \xi^{i}$. Since $\sigma \sim \partial_{t} f(t)$ the only consistent choices are $f(t)=\alpha+\beta t$ and $\nabla_{i} \xi^{i}=\frac{2}{z} \beta$, for constant $\alpha$ and $\beta$. One may further choose to set $\beta=0$ (though this is not necessary in all the cases) as in [19]. Then one has $\xi=\alpha \partial_{t}+\xi^{i} \partial_{i}$ with constant $\alpha$ and $\nabla_{i} \xi^{i}=0$, giving rise to an algebra isomorphic to $\mathbb{R} \times \mathcal{A}$ where $\mathcal{A}$ is the algebra of volume-preserving (smooth) diffeomorphisms of $a_{i j}$ (say $\mathbb{R}^{2}$ or round $S^{2}$ ). In the $z=1$ case such an algebra has appeared recently [19] in a different context.

We anticipate that it should be possible to gauge fix the background such that one obtains the extension of $\mathfrak{b m s}_{4}$ considered in [20]. We will leave further studies of these aspects to a future publication.

\section{Conclusion}

We have derived equations of motion for a scalar field coupled to a generic 3-dimensional Carroll geometry that are Carroll diffeomorphic and Weyl covariant. These exist for any

\footnotetext{
${ }^{3} \mathrm{~A}$ weaker condition is to fix $b_{i}$ to be such that $f_{i j}=0$.
} 
value of the dynamical exponent $z$ and conformal dimension $\delta$ of the scalar. We have also shown how to construct corresponding actions for such theories and analysed the consequences for $z$ and $\delta$. After an appropriate gauge fixing we found that the field theories we sought, with Carrollian conformal symmetries have in addition to $\Phi(t, \mathbf{x})$ two more dynamical fields coming from $b_{i}(t, \mathbf{x})$ in their field content. The space-like theories resemble more (deformations) of standard Euclidean CFTs with additional features than the timelike theories.

Even though we have demonstrated our methods most explicitly for 3-dimensional Carroll spacetimes the generalisation to arbitrary (higher) dimensions is straightforward - see the appendix A for some technical details of this exercise. Also one can include Carroll like Maxwell, Yang-Mills fields and other matter fields following similar methods - these details will be presented in [21].

We have concentrated on classical theories in this paper. It will be interesting to explore the quantum aspects of our theories - including renormalisation, anomalies etc. We need to compute the Noether charges for the symmetries and show that they form $\mathfrak{c c a}_{3}^{(z)}$. In particular we will need to construct the soft charges - which may lead to Ward identities of celestial amplitudes in connection with the soft graviton theorems (see [22] for a review).

There are some features expected of holographic duals of flat space gravity [23, 24]. It will be interesting to see if these are borne out in theories of the type we constructed here. In this connection, we anticipate that the existence of additional fields, either $b_{i}(t, \mathbf{x})$ (or $a_{i j}(t, \mathbf{x})$ with $a=1$ ) may play a useful role. One also expects such requirements to impose further cuts on the spaces of classical theories we find, along with any other possible quantum consistency conditions.

In this work we concentrated on Carrollian theories. However, the same techniques can be used for constructing the Galilean theories as well. There is a curious duality between Carrollian and Galilean field theories [3]. It would be interesting to check if such duality exists between our theories and the corresponding Galilean ones. We hope to present results on this in the near future.

Eventually one would like to construct fully consistent (perhaps supersymmetric) Carrollian CFTs which can potentially be useful to describe flat space gravity/string theories holographically. We hope that our methods would lead to more avenues to explore than the method of taking ultra-relativistic limits of known theories.

\section{Acknowledgments}

We thank participants of Chennai Strings Meeting 2019 (Nov 21-23) at IMSc for their feedback on a talk by NVS on this topic.

\section{A Extension to general dimensions — some details}

In this appendix we provide some technical details of the extension of the $d=2$ computations of this paper to general $d$. Let us start by providing the transformation properties of 
various Carroll diffeomorphic quantities under Carroll Weyl transformations - generalising those of (3.12).

$$
\begin{aligned}
\theta & \longrightarrow B^{z-1}\left[B \theta-d \hat{\partial}_{t} B\right], \quad \theta^{2} \longrightarrow B^{2 z-2}\left[B^{2} \theta-2 B \theta d \hat{\partial}_{t} B+d^{2}\left(\hat{\partial}_{t} B\right)^{2}\right] \\
\hat{\gamma}_{j}^{i} \hat{\gamma}_{i}^{j} & \longrightarrow B^{2 z-2}\left[B^{2} \hat{\gamma}_{j}^{i} \hat{\gamma}_{i}^{j}-2 B \theta \hat{\partial}_{t} B+d\left(\hat{\partial}_{t} B\right)^{2}\right] \\
\hat{r} & \longrightarrow B^{2} \hat{r}-d(d-1) a^{i j} \hat{\nabla}_{i} B \hat{\nabla}_{j} B+2(d-1) a^{i j} B \hat{\nabla}_{i} \hat{\nabla}_{j} B \\
a^{i j} \hat{\nabla}_{i} \phi_{j} & \longrightarrow B^{2} a^{i j} \hat{\nabla}_{i} \phi_{j}+z(d-1) a^{i j} \hat{\nabla}_{i} B \hat{\nabla}_{j} B-z B a^{i j} \hat{\nabla}_{i} \hat{\nabla}_{j} B-B(d-2) a^{i j} \phi_{i} \hat{\nabla}_{j} B \\
a^{i j} \phi_{i} \phi_{j} & \longrightarrow B^{2} a^{i j} \phi_{i} \phi_{j}-2 z B a^{i j} \phi_{i} \hat{\nabla}_{j} B+z^{2} a^{i j} \hat{\nabla}_{i} B \hat{\nabla}_{j} B
\end{aligned}
$$

The combinations that transform homogeneously are

$$
\begin{aligned}
\hat{r}+\frac{2}{z}(d-1) a^{i j} & \hat{\nabla}_{i} \phi_{j}-\frac{(d-2)(d-1)}{z^{2}} a^{i j} \phi_{i} \phi_{j} \\
& \longrightarrow B^{2}\left(\hat{r}+\frac{2}{z}(d-1) a^{i j} \hat{\nabla}_{i} \phi_{j}-\frac{(d-2)(d-1)}{z^{2}} a^{i j} \phi_{i} \phi_{j}\right) \\
\hat{\gamma}_{j}^{i} \hat{\gamma}_{i}^{j}-\frac{\theta^{2}}{d} & \longrightarrow B^{2 z}\left[\hat{\gamma}_{j}^{i} \hat{\gamma}_{i}^{j}-\frac{\theta^{2}}{d}\right]
\end{aligned}
$$

Taking that the scalar $\Phi$ transforms as $\phi \rightarrow B^{\delta} \Phi$ under Weyl transformations various expression transforms as follows

$$
\begin{aligned}
a^{i j} \phi_{i} \hat{\partial}_{j} \Phi & \longrightarrow B^{\delta}\left[B^{2} a^{i j} \phi_{j} \hat{\partial}_{i} \Phi+\delta B a^{i j} \phi_{i} \hat{\partial}_{j} B \Phi-\delta z a^{i j} \hat{\nabla}_{i} B \hat{\nabla}_{j} B \Phi-z B a^{i j} \hat{\partial}_{i} B \hat{\partial}_{j} \Phi\right] \\
\hat{\nabla}_{i} \hat{\partial}^{i} \Phi & \longrightarrow B^{\delta}\left[B^{2} \hat{\nabla}_{i} \hat{\partial}^{i} \Phi+\left(\delta^{2}-d \delta+\delta\right) a^{i j} \hat{\nabla}_{i} B \hat{\nabla}^{i} B \Phi+\delta B \hat{\nabla}_{i} \hat{\nabla}^{i} B \Phi+(2+2 \delta-d) B \hat{\partial}_{i} B \hat{\partial}^{i} \Phi\right]
\end{aligned}
$$

The simplest Weyl covariant object at first order derivative is

$$
\hat{\partial}_{t} \Phi+\frac{\delta}{d} \theta \Phi \longrightarrow B^{z+\delta}\left(\hat{\partial}_{t} \Phi+\frac{\delta}{d} \theta \Phi\right)
$$

At second order we find:

$$
\begin{aligned}
& \hat{\partial}_{t}^{2} \Phi+\frac{1}{d}(2 \delta+z) \theta \hat{\partial}_{t} \Phi+\frac{\delta}{d}\left[\frac{1}{d}(z+\delta) \theta^{2}+\hat{\partial}_{t} \theta\right] \Phi \\
& \longrightarrow B^{2 z+\delta}\left(\hat{\partial}_{t}^{2}+\frac{1}{d}(2 \delta+z) \theta \hat{\partial}_{t} \Phi+\frac{\delta}{d}\left[\frac{1}{d}(z+\delta) \theta^{2}+\hat{\partial}_{t} \theta \Phi\right]\right) \\
& \hat{\nabla}^{i} \hat{\partial}_{i} \Phi+\frac{(2-d+2 \delta)}{z} \phi^{i} \hat{\partial}_{i} \Phi-\frac{\delta}{2}\left[\frac{1}{d-1} \hat{r}-\frac{1}{z^{2}}(2-d+2 \delta) \phi^{i} \phi_{i}\right] \Phi \\
& \longrightarrow B^{2+\delta}\left(\hat{\nabla}^{i} \hat{\partial}_{i} \Phi+\frac{(2-d+2 \delta)}{z} \phi^{i} \hat{\partial}_{i} \Phi-\frac{\delta}{2}\left[\frac{1}{d-1} \hat{r}-\frac{1}{z^{2}}(2-d+2 \delta) \phi^{i} \phi_{i}\right] \Phi\right)
\end{aligned}
$$


For $z=1$ the covariant equation can be written as,

$$
\begin{aligned}
& \kappa_{0}\left[\hat{\partial}_{t}^{2} \Phi+\frac{1}{d}(2 \delta+z) \theta \hat{\partial}_{t} \Phi+\frac{\delta}{d}\left[\frac{1}{d}(z+\delta) \theta^{2}+\hat{\partial}_{t} \theta\right] \Phi\right] \\
& +\kappa_{1}\left[\hat{\nabla}^{i} \hat{\partial}_{i} \Phi+\frac{(2-d+2 \delta)}{z} \phi^{i} \hat{\partial}_{i} \Phi-\frac{\delta}{2}\left[\frac{1}{d-1} \hat{r}-\frac{1}{z^{2}}(2-d+2 \delta) \phi^{i} \phi_{i}\right] \Phi\right] \\
& +\left[\sigma_{0}\left(\hat{\gamma}_{j}^{i} \hat{\gamma}_{i}^{j}-\frac{\theta^{2}}{d}\right)+\sigma_{1}\left(\hat{r}+\frac{2}{z}(d-1) a^{i j} \hat{\nabla}_{i} \phi_{j}-\frac{(d-2)(d-1)}{z^{2}} a^{i j} \phi_{i} \phi_{j}\right)+\sigma_{2} f_{i j} f^{i j}\right] \Phi+\lambda \Phi \frac{2+\delta}{\delta}=0
\end{aligned}
$$

The general $d$ expressions of invariants in (3.27)-(3.31) that can be used to construct actions are

$$
\begin{aligned}
& \Phi\left(\hat{\partial}_{t} \Phi+\frac{\delta}{d} \theta \Phi\right) \\
& \left(\hat{\partial}_{t} \theta+\frac{z}{2} \hat{\gamma}_{j}^{i} \hat{\gamma}_{i}^{j}\right) \Phi^{2}+\frac{d}{\delta} \Phi \hat{\partial}_{t}^{2} \Phi-\frac{d(z+2 \delta)}{2 \delta^{2}}\left(\hat{\partial}_{t} \Phi\right)^{2} \\
& \left(\hat{\partial}_{t} \Phi\right)^{2}+\delta \theta \Phi \hat{\partial}_{t} \Phi+\frac{\delta^{2}}{d} \gamma_{j}^{i} \hat{\gamma}_{i}^{j} \Phi^{2}
\end{aligned}
$$

At second order in space derivatives there are three more combinations with Weyl weight $2+2 \delta$ :

$$
\begin{aligned}
& {\left[\hat{r}+\frac{2}{z}(d-1) a^{i j} \hat{\nabla}_{i} \phi_{j}-\frac{(d-2)(d-1)}{z^{2}} a^{i j} \phi_{i} \phi_{j}\right] \Phi^{2}} \\
& \hat{\partial}_{i} \Phi \hat{\partial}^{i} \Phi+2 \frac{\delta}{z} \Phi \phi^{i} \hat{\partial}_{i} \Phi+\frac{\delta^{2}}{z^{2}} \phi^{i} \phi_{i} \Phi^{2} \\
& \Phi \hat{\nabla}^{i} \hat{\partial}_{i} \Phi-\frac{d-2(1+\delta)}{z} \Phi \phi^{i} \hat{\partial}_{i} \Phi+\frac{\delta}{z} \hat{\nabla}^{i} \phi_{j} \Phi^{2}+\frac{\delta}{z^{2}}(2-d+\delta) \phi^{i} \phi_{i} \Phi^{2}
\end{aligned}
$$

These can be used to construct Carroll diffeomorphic and Weyl invariant actions for general $d$ straightforwardly.

Open Access. This article is distributed under the terms of the Creative Commons Attribution License (CC-BY 4.0), which permits any use, distribution and reproduction in any medium, provided the original author(s) and source are credited.

\section{References}

[1] J-M. Levy-Leblond, Une nouvelle limite non-relativiste du group de Poincare, Ann. Inst. Henri Poincare 3 (1965) 1.

[2] N.D. Sen Gupta, On an analogue of the Galilei group, Nuovo Cim. 44 (1966) 512.

[3] C. Duval, G.W. Gibbons, P.A. Horvathy and P.M. Zhang, Carroll versus Newton and Galilei: two dual non-Einsteinian concepts of time, Class. Quant. Grav. 31 (2014) 085016 [arXiv: 1402.0657] [INSPIRE].

[4] C. Duval, G.W. Gibbons and P.A. Horvathy, Conformal Carroll groups, J. Phys. A 47 (2014) 335204 [arXiv:1403.4213] [INSPIRE]. 
[5] C. Duval, G.W. Gibbons and P.A. Horvathy, Conformal Carroll groups and BMS symmetry, Class. Quant. Grav. 31 (2014) 092001 [arXiv: 1402.5894] [INSPIRE].

[6] M. Henneaux, Geometry of Zero Signature Space-times, Bull. Soc. Math. Belg. 31 (1979) 47.

[7] H. Bondi, M.G.J. van der Burg and A.W.K. Metzner, Gravitational waves in general relativity. 7. Waves from axisymmetric isolated systems, Proc. Roy. Soc. Lond. A 269 (1962) 21.

[8] R. Sachs, Asymptotic symmetries in gravitational theory, Phys. Rev. 128 (1962) 2851 [INSPIRE].

[9] G. Barnich and C. Troessaert, Aspects of the BMS/CFT correspondence, JHEP 05 (2010) 062 [arXiv: 1001.1541 ] [INSPIRE].

[10] G. Barnich and C. Troessaert, Symmetries of asymptotically flat 4 dimensional spacetimes at null infinity revisited, Phys. Rev. Lett. 105 (2010) 111103 [arXiv:0909.2617] [INSPIRE].

[11] L. Ciambelli, R.G. Leigh, C. Marteau and P.M. Petropoulos, Carroll Structures, Null Geometry and Conformal Isometries, Phys. Rev. D 100 (2019) 046010 [arXiv:1905.02221] [INSPIRE].

[12] L. Ciambelli, C. Marteau, A.C. Petkou, P.M. Petropoulos and K. Siampos, Flat holography and Carrollian fluids, JHEP 07 (2018) 165 [arXiv:1802.06809] [INSPIRE].

[13] L. Ciambelli, C. Marteau, A.C. Petkou, P.M. Petropoulos and K. Siampos, Covariant Galilean versus Carrollian hydrodynamics from relativistic fluids, Class. Quant. Grav. 35 (2018) 165001 [arXiv: 1802.05286] [INSPIRE].

[14] A. Bagchi, R. Basu, A. Mehra and P. Nandi, Field Theories on Null Manifolds, JHEP 02 (2020) 141 [arXiv : 1912.09388] [INSPIRE].

[15] A. Bagchi, A. Mehra and P. Nandi, Field Theories with Conformal Carrollian Symmetry, JHEP 05 (2019) 108 [arXiv:1901.10147] [INSPIRE].

[16] A. Bagchi, R. Basu, A. Kakkar and A. Mehra, Flat Holography: Aspects of the dual field theory, JHEP 12 (2016) 147 [arXiv:1609. 06203] [INSPIRE].

[17] L. Ciambelli and C. Marteau, Carrollian conservation laws and Ricci-flat gravity, Class. Quant. Grav. 36 (2019) 085004 [arXiv:1810.11037] [INSPIRE].

[18] R.M. Wald, General Relativity, University of Chicago Press, (1984) [DOI].

[19] G. Compère, A. Fiorucci and R. Ruzziconi, The $\Lambda-B M S_{4}$ group of $d S_{4}$ and new boundary conditions for $A d S_{4}$, Class. Quant. Grav. 36 (2019) 195017 [arXiv: 1905.00971] [inSPIRE].

[20] M. Campiglia and A. Laddha, Asymptotic symmetries and subleading soft graviton theorem, Phys. Rev. D 90 (2014) 124028 [arXiv:1408.2228] [INSPIRE].

[21] N. Gupta and N.V. Suryanarayana, work in progress.

[22] A. Strominger, Lectures on the Infrared Structure of Gravity and Gauge Theory, arXiv: 1703.05448 [INSPIRE].

[23] S. Banerjee, P. Pandey and P. Paul, Conformal properties of soft operators: Use of null states, Phys. Rev. D 101 (2020) 106014 [arXiv:1902.02309] [inSPIRE].

[24] S. Banerjee and P. Pandey, Conformal properties of soft-operators. Part II. Use of null-states, JHEP 02 (2020) 067 [arXiv: 1906. 01650] [INSPIRE]. 